\title{
A freestanding CNTs film fabricated by pyrrole-modified CVD for capacitive deionization
}

\author{
Dan Song BE \\ Key Laboratory of Advanced Ceramics and Machining Technology of the \\ Ministry of Education, School of Materials Science and Engineering, Tianjin \\ University, Tianjin, China \\ Wenlei Guo MSc \\ Key Laboratory of Advanced Ceramics and Machining Technology of the \\ Ministry of Education, School of Materials Science and Engineering, Tianjin \\ University, Tianjin, China

\section{Tao Zhang BE} \\ Key Laboratory of Advanced Ceramics and Machining Technology of the \\ Ministry of Education, School of Materials Science and Engineering, Tianjin \\ University, Tianjin, China

\section{Pengyi Lu BE} \\ Key Laboratory of Advanced Ceramics and Machining Technology of the \\ Ministry of Education, School of Materials Science and Engineering, Tianjin \\ University, Tianjin, China
}

\author{
Anran Guo PhD \\ Key Laboratory of Advanced Ceramics and Machining Technology of the \\ Ministry of Education, School of Materials Science and Engineering, Tianjin \\ University, Tianjin, China \\ Feng Hou PhD \\ Key Laboratory of Advanced Ceramics and Machining Technology of the \\ Ministry of Education, School of Materials Science and Engineering, Tianjin \\ University, Tianjin, China (corresponding author: houf@tju.edu.au) \\ Xiao Yan PhD \\ Guangdong Key Laboratory of Membrane Materials and Membrane \\ Separation, Guangzhou Institute of Advanced Technology, Chinese \\ Academy of Sciences, Guangzhou, China \\ Ji Liang PhD \\ Institute for Superconducting and Electronic Materials, Australian Institute \\ of Innovative Materials, University of Wollongong, Innovation Campus, \\ Wollongong, Australia
}

Carbon nanotubes (CNTs) are an excellent electrode material for capacitive deionization (CDI), due to their excellent electronic conductivity and outstanding chemical/physical stability. Their powder form and easy aggregation, however, have greatly limited their practical CDI performance. Aiming to address this issue, the authors report a freestanding CNT film which was fabricated by floating-catalyst chemical vapor deposition, as a binder-free electrode for CDI. By simply adjusting the pyrrole content in the precursor, the morphology of the resulting CNT film can be tuned to meet the requirements of CDI. In the presence of $2 \mathrm{wt} \%$ pyrrole, the CNT film with a mesoporous structure exhibited a large specific surface area of $198 \mathrm{~m}^{2} / \mathrm{g}$ and an increased electric double-layer capacity $(40 \mathrm{~F} / \mathrm{g})$, which is more than two times as large as that of the pristine CNT film. Due to these merits, the electrosorption capacity for sodium chloride $(\mathrm{NaCl})$ of the CNT film electrode $(11.39 \mathrm{mg} / \mathrm{g})$ has been greatly improved compared with that of the pristine CNT film $(4.52 \mathrm{mg} / \mathrm{g})$, showing a good potential for large-scale practical CDI.

\section{Notation}

$C_{0} \quad$ initial sodium chloride $(\mathrm{NaCl})$ concentration

$C_{\mathrm{m}} \quad$ specific capacitance

$C_{\mathrm{e}} \quad$ final sodium chloride concentrations

$\mathrm{d} V \quad$ volume of the pore

I charge-discharge current

$I_{\mathrm{D}} \quad$ intensity of $\mathrm{D}$ band

$I_{\mathrm{G}} \quad$ intensity of $\mathrm{G}$ band

$m \quad$ mass of the active materials

$P \quad$ partial pressure of nitrogen

$P_{0} \quad$ saturated vapor pressure of liquid nitrogen at the specific temperature

$q_{\mathrm{e}} \quad$ electrosorption capacity

$R_{\mathrm{ct}} \quad$ charge-transfer resistance

$R_{\mathrm{S}} \quad$ series resistance

$r \quad$ radius of the pore

$V \quad$ volume of the sodium chloride solution

$\Delta t \quad$ discharge time

$\Delta V \quad$ discharge potential window

\section{Introduction}

The exploding growth of population and rapid industrialization have raised a series of severe environmental issues to the society.
Consequently, the sustainability of essential resources, such as fresh water, has attracted an increasing amount of concern, particularly considering that seawater and brackish water account for a large portion of the total water volume on earth. ${ }^{1,2}$ Therefore, developing efficient desalination technologies to remove salts/ions from seawater/brackish water is in urgent demand to acquire fresh water from these resources. ${ }^{3}$

Traditional desalination technologies, including membrane separation, thermal separation, reverse osmosis, multistage flash distillation and electrodialysis, ${ }^{1}$ have their intrinsic shortcomings, such as high energy consumption, high maintenance costs and significant regeneration difficulties. ${ }^{4-6}$ In comparison, capacitive deionization (CDI), a recently emerged technology for removing cations/anions in water by electrostatic force, has become a hot research topic due to its multiple merits, including high energy effectiveness, simple regeneration, no secondary pollution and low cost. ${ }^{7}$ The CDI process basically consists of two stages: $(a)$ the ion adsorption stage, in which the ions are absorbed on the positive/negative electrodes when a voltage is applied, and $(b)$ the release of ions upon the removal of the voltage or application of a reversed voltage. ${ }^{8}$ In a CDI device, the electrode is the most essential component, which typically requires a high specific 
surface area, good electrical conductivity and manageable pore structure to achieve high CDI performance. ${ }^{9}$

Carbon (C)-based materials, such as activated carbon (AC), ${ }^{10,11}$ AC nanofibers, ${ }^{12}$ carbon aerogel, ${ }^{13}$ carbon nanotubes $(\mathrm{CNTs})^{7,14,15}$ and graphene, ${ }^{16,17}$ have been exploited for CDI. In particular, CNTs, with a unique one-dimensional tubular nanostructure, are particularly suitable for CDI, due to their superior structural/ compositional properties, such as suitable pore structure, good physical/chemical stability and excellent conductivity. ${ }^{18,19}$ For example, Dai et al. ${ }^{20}$ explored the relationship between the specific surface area and CDI capacity of three kinds of multiwalled CNTs, including unpurified CNTs, purified CNTs and graphitized CNTs. The result indicated that the CDI capacity is proportional to the specific surface area of CNTs, which is essentially determined by multiple parameters, namely, the fabrication temperature, carrier gas flow velocity and atmosphere, during the chemical vapor deposition (CVD) process ${ }^{21,22}$ and the subsequent treatment of CNTs such as purification and carbonization. ${ }^{20}$

By far, the actual CDI performance of reported CNT-based electrodes is unsatisfactory, which is mainly caused by the use of binder for the powder-form CNTs to decrease the specific surface area and deteriorate the pore structure of the obtained electrode. ${ }^{20,23,24}$ Specifically, a conventional CNT-based electrode is fabricated by directly compressing or slurry coating of a mixture of CNT powder, binder and conductive agent on a current collector. ${ }^{25}$ In this process, the binders/conductive agents usually lead to severe aggregation of CNTs, block the pores between individual CNTs and consequently hinder the diffusion of ions. ${ }^{24}$ Therefore, the development of binder-free CNT-based electrodes with good electronic conductivity and suitable porosity will greatly improve their practical CDI performance.

Herein, aiming to address the aforementioned issues, the authors report a binder-free and continuous CNT film electrode, which was prepared by the using the floating-catalyst chemical vapor deposition (FCCVD) method. By introducing pyrrole into the precursor solution, the morphology of CNTs can be adjusted and optimized. With $2 \mathrm{wt} \%$ of pyrrole in the precursor, a large specific surface area of $198 \mathrm{~m}^{2} / \mathrm{g}$ and a mesoporous structure have been achieved for the obtained CNT film, doubling the electric double-layer capacitance compared to that of the pristine CNT film. Due to these merits, a high CDI capacity of $11.39 \mathrm{mg} / \mathrm{g}$ has been achieved for the obtained CNT film, showing the great potential of this material for CDI.

\section{Experiment}

\subsection{Synthesis of CNT film}

The CNT films were fabricated by using the FCCVD method. ${ }^{20}$ In a typical fabrication, $0.2 \mathrm{~g}$ thiophene $\left(\mathrm{C}_{4} \mathrm{H}_{4} \mathrm{~S} ; 1.0 \mathrm{wt} . \%\right.$, analytical reagent (AR) grade, Tianjin Guangfu Fine Chemical Research Institute) and $0.375 \mathrm{~g}$ ferrocene $\left(\mathrm{C}_{10} \mathrm{H}_{10} \mathrm{Fe} ; 1.9 \mathrm{wt} . \%\right.$, AR grade, Tianjin Guangfu Fine Chemical Research Institute) were first dispersed in $25 \mathrm{ml}$ ethanol $\left(\mathrm{C}_{2} \mathrm{H}_{5} \mathrm{OH} ; 98 \%\right.$, guaranteed reagent grade, Tianjin Jiangtian Chemical Technology Co. Ltd). The mixture precursor solution was then treated with ultrasonication for $40 \mathrm{~min}$ at $50^{\circ} \mathrm{C}$. Subsequently, the precursor was injected into the tube of the vertical furnace $\left(1150^{\circ} \mathrm{C}\right)$ from the top at the rate of $8 \mathrm{ml} / \mathrm{h}$. With a hydrogen gas $\left(\mathrm{H}_{2}\right)$ flow $(800$ standard $\mathrm{cm}^{3} / \mathrm{min}$ ) downstream in the furnace tube, thin film was gathered at the bottom of the reactor. By varying the pyrrole content in the precursor solution, CNT films with various structures were prepared. The obtained CNT films were named as CNTs-a, CNTs-b and CNTs-c, corresponding to pyrrole contents of 0,1 and $2 \mathrm{wt} . \%$ in the precursor, respectively.

\subsection{Material characterization}

The morphology of the CNT films was examined by scanning electron microscopy (SEM; Hitachi, S-4800) at an operating voltage of $10 \mathrm{kV}$, and by transmission electron microscopy (TEM, Jem-2100) at an acceleration voltage of $200 \mathrm{kV}$. The X-ray diffraction (XRD) patterns of the CNT films were obtained using a D8 Advance diffractometer with a copper $(\mathrm{Cu}) \mathrm{K} \alpha$ radiation source at room temperature. The Raman spectra of the materials were recorded by using a laser Raman spectrometer (UK, Renishaw) using $532 \mathrm{~nm}$ laser. Nitrogen $(\mathrm{N})$ adsorption and desorption isotherms were collected on an Asap 2020 system (Micromeritics) at $77 \mathrm{~K}$. All the studies of the electrochemical properties, including cyclic voltammetry (CV), galvanostatic charging-discharging (GCD) and electrochemical impedance spectroscopy (EIS), were performed using an electrochemical workstation (CHI 660D, Shanghai Chenhua Co.) on a three-electrode testing system (counter electrode: platinum $(\mathrm{Pt})$ sheet; reference electrode: mercury $(\mathrm{Hg}) /$ mercury (II) oxide $(\mathrm{HgO})$ ) in $6 \mathrm{M}$ potassium hydroxide $(\mathrm{KOH})$ aqueous solution. The as-prepared CNT films were pressed onto nickel foam under a pressure of $10 \mathrm{MPa}$ for $20 \mathrm{~s}$ to prepare the working electrode. The specific capacitance $\left(C_{\mathrm{m}}\right)$ was calculated according to the following equation

1. $C_{\mathrm{m}}=\frac{I \Delta t}{m \Delta V}$

where $I$ is the charge-discharge current, $\Delta t$ is the discharge time, $\Delta V$ is the discharge potential window and $m$ is the mass of the active materials.

The adsorption performance of the CNT films was tested by a selfmade CDI device (Figure 1). The sodium chloride $(\mathrm{NaCl})$ aqueous solution $(\sim 100 \mu \mathrm{S} / \mathrm{cm}, 40 \mathrm{ml})$ flows into the CDI unit by a peristaltic pump through the inlet at the bottom of the CDI unit (flow rate: $10 \mathrm{ml} / \mathrm{min}$ ). The voltage used in the experiment was $2 \cdot 0 \mathrm{~V}$ by a direct-current (DC) power. Furthermore, the change in conductivity during the measurement was continuously recorded using a conductivity monitor (DDS-307F) and the relationship between the conductivity and the solution concentration was calibrated before the experiment. Herein, the electrosorption capacity $\left(q_{\mathrm{e}}, \mathrm{mg} / \mathrm{g}\right)$ of the electrode was calculated using the following formula 


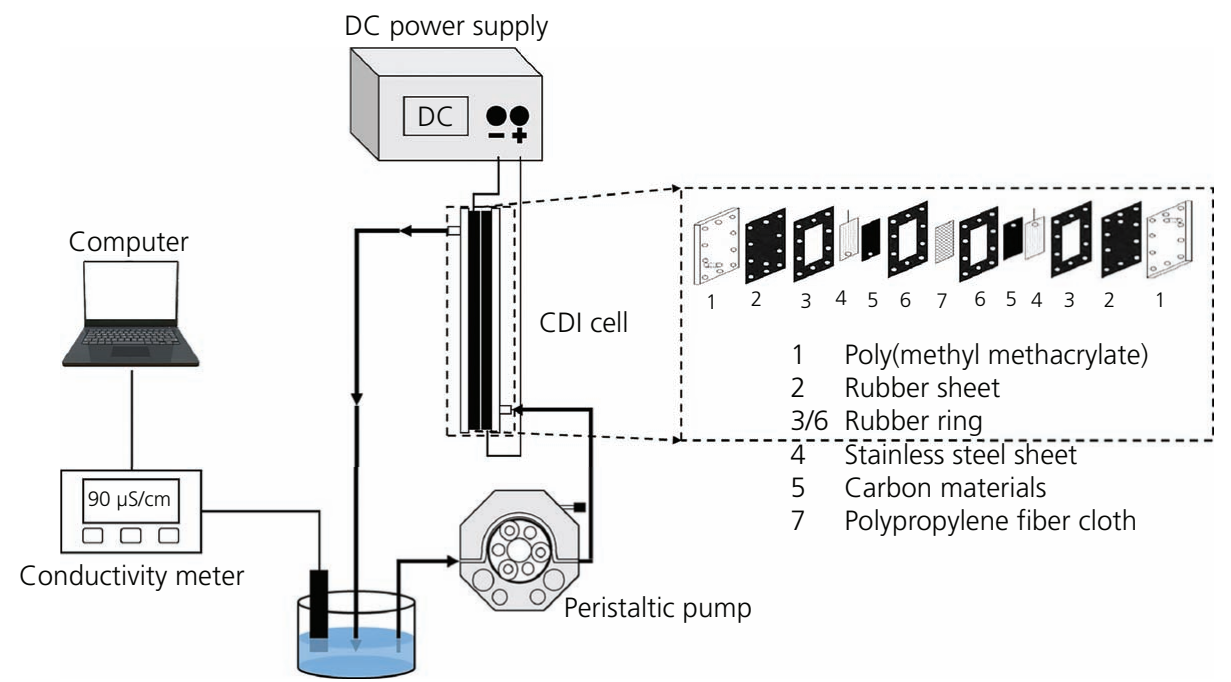

Figure 1. Schematic diagram of measurements on the electrosorptive capacity of the CNT films

2. $q_{\mathrm{e}}=\frac{\left(C_{\mathrm{e}}-C_{0}\right) V}{m}$

where $C_{0}(\mathrm{mg} / \mathrm{ml})$ and $C_{\mathrm{e}}(\mathrm{mg} / \mathrm{ml})$ are the initial and final sodium chloride concentrations, respectively; $V(\mathrm{ml})$ is the volume of the sodium chloride solution; and $m(\mathrm{~g})$ is the total mass of the two working electrodes.

\section{Results and discussion}

The CNT film was fabricated by using the apparatus shown in Figure 2(a). The precursor solution, containing ethanol, ferrocene, thiophene and/or pyrrole, was directly injected into the vertical reaction chamber and carried into the hot zone $\left(1150^{\circ} \mathrm{C}\right)$ by the hydrogen carrier gas. The precursor decomposed under the high temperature and formed an aerogel of CNTs, which transformed into CNT film when carried downward by the hydrogen gas to the bottom of the chamber. Finally, the CNT film was collected on the spindle beneath the chamber (Figure 2(b)). It can be easily unrolled from the spindle, showing good flexibility (Figure 2(c)). Typically, a single piece of the unfolded film can reach $15 \times 12 \times$ $0.01 \mathrm{~cm}^{3}$ (Figure 2(d)), which was fabricated at a spindle rotating speed of 60 revolutions/min and a collection time of $20 \mathrm{~min}$. The as-fabricated CNT film exhibits good mechanical strength and can be twisted into various shapes (Figure 2(e)). Noticeably, the introduction of pyrrole ( 1 or $2 \mathrm{wt.} \%$ ) in the precursor will not affect the apparent mechanical behavior of the obtained film.

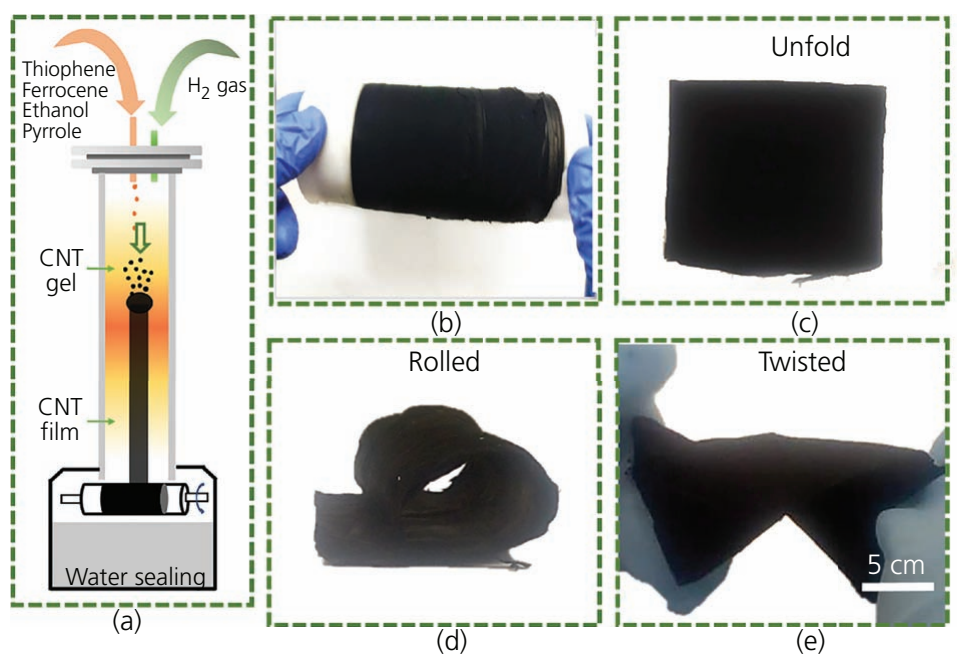

Figure 2. (a) Schematic illustration of the preparation of CNT film; (b-e) the proof of the CNT film's flexibility 

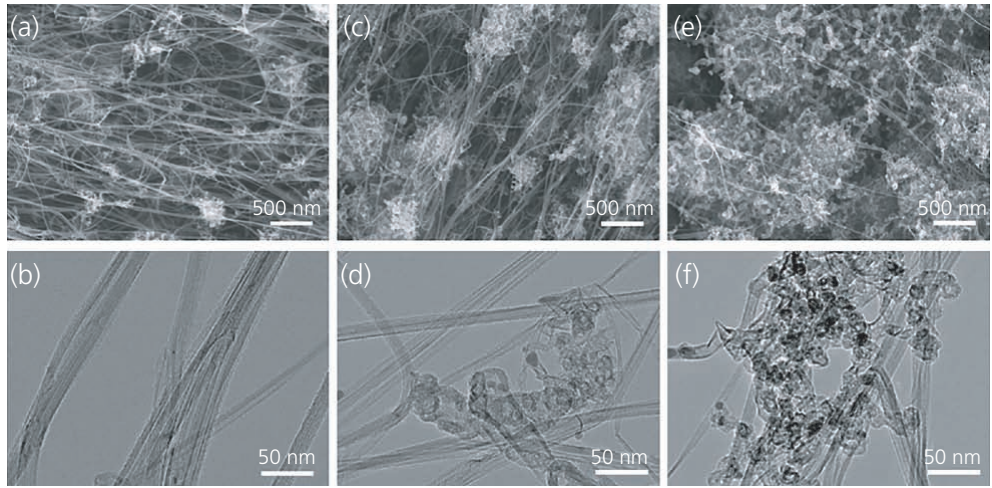

Figure 3. SEM images of (a) CNTs-a, (c) CNTs-b and (e) CNTs-c; TEM images of (b) CNTs-a, (d) CNTs-b and (f) CNTs-c

The morphology and microstructure of the obtained CNT films were firstly investigated by SEM and TEM (Figure 3). The pristine CNTs-a shows a uniform network of entangled CNT bundles ( 20 nm, Figures 3(a) and 3(b)). Typically, most of the CNT bundles in the CNTs-a film are long and straight, with some CNTs stretching out to connect with other bundles and form a network. Upon the introduction of pyrrole in the precursor, the obtained CNTs-a films (i.e. CNTs-b and CNTs-c) possessed a distinct structural and morphological change compared with the pristine CNTs-a. Compared with CNTs-a, pyrrole led to the formation of curved CNTs (Figure 3(c)), which are randomly distributed throughout the CNT network or between the CNTs or are attached to the long straight CNTs. According to previous studies, the pyrolysis of pyrrole releases free nitrogen atoms, which may promote the formation of five- or seven-membered carbon rings and cause the curving of CNTs (Figure $3(\mathrm{~d})$ ). ${ }^{26}$ At a higher pyrrole concentration of $2 \mathrm{wt} \%$ in the precursor (i.e. CNTs-c), the structural change was more obvious (Figure 3(e)). In this material, the content of long and straight CNTs with a small diameter was substantially reduced, while a large amount of curled CNTs with a relatively larger diameter $(c .40 \mathrm{~nm})$ appeared. Moreover, these curled CNTs are uniformly dispersed in the view and have been the main constituent unit of the CNTs-c film. On the contrary, the amount of the long straight CNTs, which takes a large portion in CNTs-a and CNTs-b, became much less in this material (Figure 3(f)).

XRD was then utilized to study the crystal structure of the asprepared materials (Figure 4(a)). The samples show a distinct peak at $26^{\circ}$, corresponding to the graphitic (002) facet of CNTs. Moreover, another peak at $44.7^{\circ}$ can also be clearly seen, from to the (110) facet of CNTs. ${ }^{27}$ It is worth noting that the full width at half maximum at $26^{\circ}$ of the CNT films (i.e. CNTs-a, CNTs-b and CNTs-c) gradually increases with an increase in the pyrrole content, which indicates that CNTs-c with a large number of curved tubes has lower crystallinity compared with CNTs-a and CNTs-b - that is, the presence of pyrrole does not introduce any extra components, but only changes the morphology and the crystallinity of the CNTs.

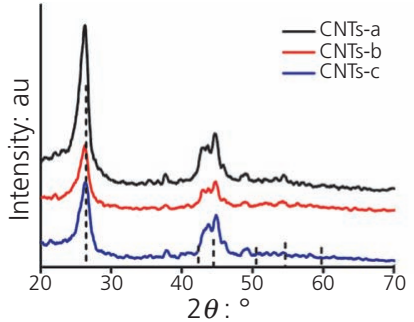

(a)

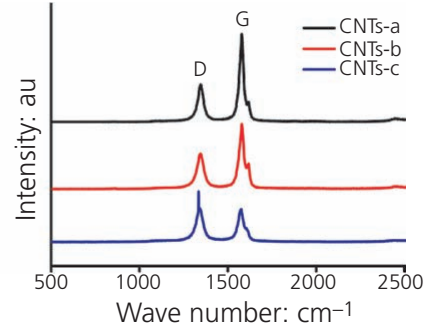

(b)
Figure 4. (a) XRD pattern of CNTs-a, CNTs-b and CNTs-c; (b) Raman spectra of the CNTs-a, CNTs-b and CNTs-c

To investigate better the structural features of the as-synthesized CNT films, the Raman spectra of the samples were also collected (Figure 4(b)). Two intensive peaks at $\sim 1351$ (D band) and $\sim 1585 \mathrm{~cm}^{-1}$ (G band) ${ }^{21}$ can be found on all materials, which are characteristic of the $\mathrm{sp}^{3}$-hybridized carbon atoms on the defective sites ( $\mathrm{D}$ band) and the $\mathrm{sp}^{2}$-hybridized carbon atoms in the graphitic framework, respectively. ${ }^{28}$ The $I_{\mathrm{D}} / I_{\mathrm{G}}$ ratios of CNTs-a, CNTs-b and CNTs-c films, which represent the magnitude of the defect in the CNT films, are $\sim 0.6, \sim 0.72$ and $\sim 1 \cdot 23$, respectively, indicating that the graphitic degree gradually decreases with the introduction of pyrrole in the precursor. This is in agreement with the aforementioned XRD results, as well as the morphological/ structural investigations of the materials as shown in Figure $3{ }^{29}$

X-ray photoelectron spectroscopy (XPS) was then employed to study the surface composition of the CNT films, as shown in Figure 5. From Figure 5(a), all the samples show the presence of only carbon $1 \mathrm{~s}$ and oxygen (O) $1 \mathrm{~s}$ peaks, at $\sim 283$ and $533 \mathrm{eV}$, respectively, and the contents of oxygen in the materials are fairly low (1.74-3.78 wt.\%). Interestingly, it is worth noting that no nitrogen signals were detected. This might be attributed to the etching of nitrogen from carbon frameworks at the high temperature $\left(1150^{\circ} \mathrm{C}\right)$, which has also been observed in other previous studies. ${ }^{30-34}$ From the high-resolution carbon 1s spectrum of CNTs-a (Figure 5(b)), the fitted peaks appear at 


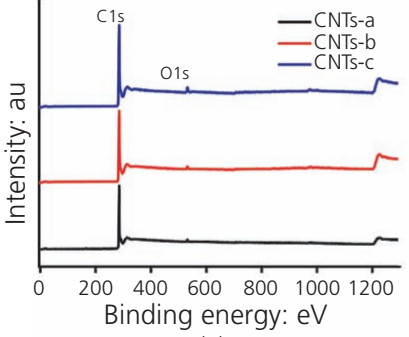

(a)

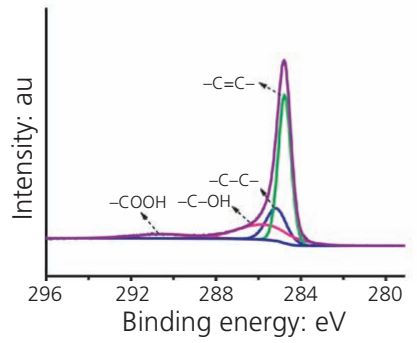

(c)

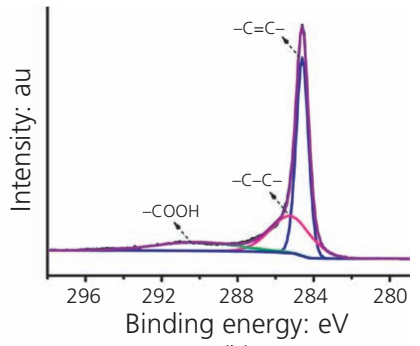

(b)

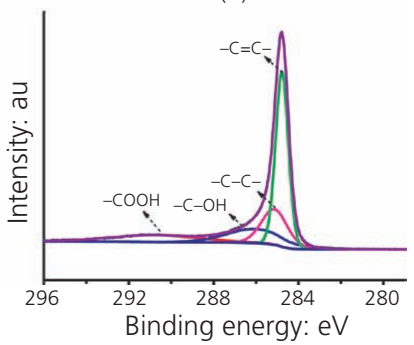

(d)
Figure 5. (a) Survey scans of the XPS spectra; XPS carbon 1s core level spectra of (b) CNTs-a, (c) CNTs-b and (d) CNTs-c

$284 \cdot 6,285 \cdot 2$ and $290 \cdot 1 \mathrm{eV}$, corresponding to the functional groups $-\mathrm{C}=\mathrm{C}-,-\mathrm{C}-\mathrm{C}-$ and $-\mathrm{COOH}$, respectively. For $\mathrm{CNTs}-\mathrm{b}$ (Figure 5(c)) and CNTs-c (Figure 5(d)), carbon 1s can be further deconvoluted into four species, the two peaks centered at $284 \cdot 6 \pm$ $0 \cdot 2$ and $285 \cdot 2 \pm 0 \cdot 2 \mathrm{eV}$ are typically ascribed to $-\mathrm{C}=\mathrm{C}-$ and $-\mathrm{C}-\mathrm{C}-$ and the other two peaks at $286 \cdot 2 \pm 0 \cdot 2$ and $290 \pm 0 \cdot 2 \mathrm{eV}$ are characteristic of the $-\mathrm{C}-\mathrm{OH}$ and $-\mathrm{COOH}$ species. ${ }^{35,36}$ Compared to the pristine $\mathrm{CNTs}$, additional the $-\mathrm{C}-\mathrm{OH}$ functional group appeared with the introduction of pyrrole and the total amount of oxygen-containing functional group $(-\mathrm{C}-\mathrm{OH}$ and $-\mathrm{COOH})$ also increased due to the influence of pyrrole, resulting in increased specific surface area and improved CDI performance.

The surface area and porosity of the materials, which have a significant influence on their CDI behavior, were further investigated by nitrogen adsorption-desorption technology (Figure 6). All the CNT films present H3-type isotherms with hysteresis loops in medium- to high-pressure regions $\left(P / P_{0}=\right.$ $0 \cdot 45-1 \cdot 0$ ), indicating the presence of both macro- and mesopores on the CNT films. ${ }^{37}$ The specific surface area of the materials was then calculated based on the Brunauer-Emmett-Teller model. CNTs-a, CNTs-b and CNTs-c possess specific surface areas of 117,176 and $198 \mathrm{~m}^{2} / \mathrm{g}$, respectively. Subsequently, the pore size distributions of three materials were obtained based on the isotherms (Figure 6 insets). For all the samples, a dominant peak appeared around $18 \mathrm{~nm}$, indicating the existence of large mesopores on the samples. Furthermore, with an increase in pyrrole content, the pore volume $\left(0.414,0.461\right.$ and $0.489 \mathrm{~cm}^{3} / \mathrm{g}$, corresponding to CNTs-a, CNTs-b and CNTs-c, respectively) of the CNT films gradually increased, due to the increase in the curved tubes. The increased surface area and enlarged pore volume, which come with the introduction of pyrrole in the precursor, would be very favorable for CDI performance by providing a large adsorption area and faster ion transport. ${ }^{38}$

Afterward, the materials' performance for CDI was firstly assessed by $\mathrm{CV}$ by directly using the films as the working electrode in a $6 \mathrm{M}$ potassium hydroxide solution in a potential region of -1 to $0 \mathrm{~V}$ against a mercury/mercury (II) oxide electrode (Figure 7). The CV loops of the three CNT film electrodes all showed a quasi-rectangular shape, indicating that the ion adsorption behavior of these materials is according to a quasielectric double-layer manner. ${ }^{39}$ Furthermore, both the CNTs-b and CNTs-c materials have a higher specific capacitance than CNTs-a at all scan rates, indicating a higher deionization capacity for removing more salt ions during $\mathrm{CDI}^{40}{ }^{40}$ Figure $7(\mathrm{~b})$ shows the GCD curves of the CNT films at a current density of $1 \cdot 0 \mathrm{~A} / \mathrm{g}$. Clearly, the charge-discharge curves are nearly linear over the whole potential range, further confirming that the CNT films exhibit an electric double-layer capacitor behavior. ${ }^{39}$ According to Equation 1, the calculated specific capacitance of CNTs-c $(40 \mathrm{~F} / \mathrm{g})$ is much larger than those of CNTs-a $(15.8 \mathrm{~F} / \mathrm{g})$ and CNTs-b $(17 \cdot 3 \mathrm{~F} / \mathrm{g})$, because the discharge time of the CNTs-c electrode is obviously longer than those of the other two electrodes. The specific capacitance of the CNTs-c film electrode is about 2.5 times that of CNTs-a and about $2 \cdot 3$ times of CNTs-b, thus suggesting its better CDI performance, which can be reasonably

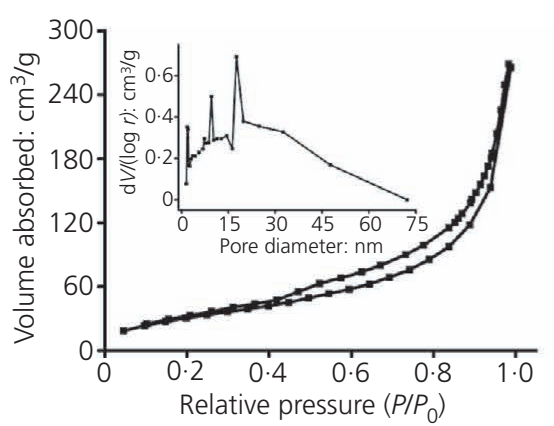

(a)

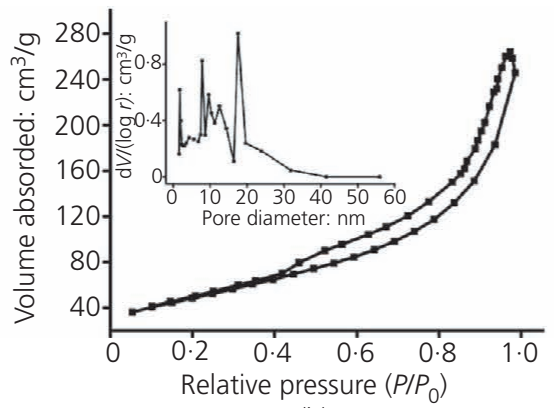

(b)

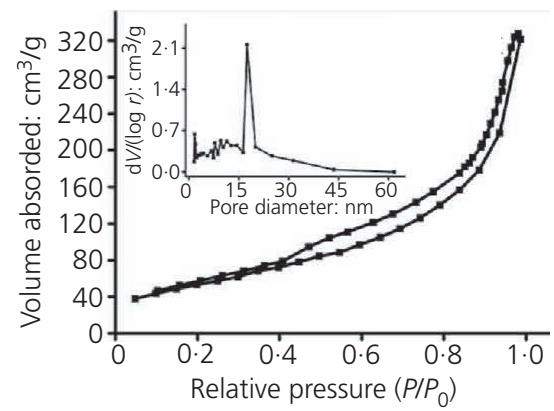

(c)

Figure 6. Nitrogen adsorption-desorption isotherms and pore size distributions (insets) of (a) CNTs-a, (b) CNTs-b and (c) CNTs-c 


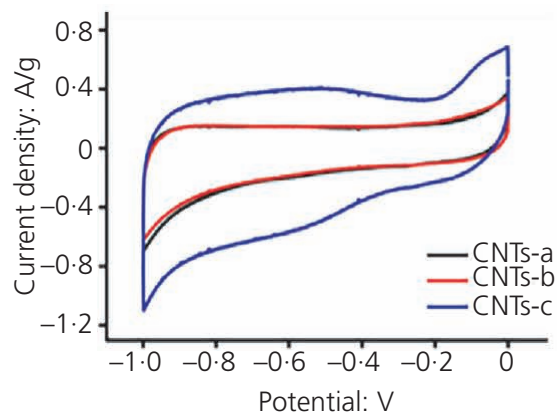

(a)

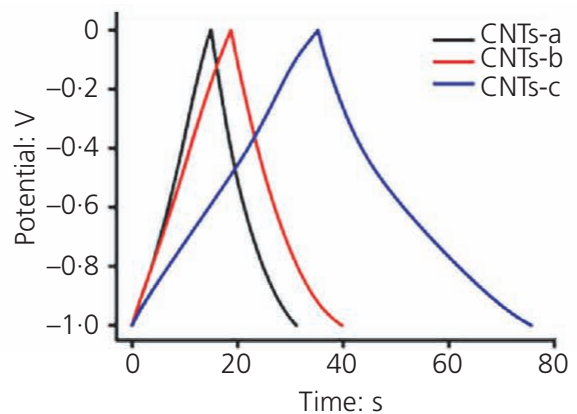

(b)

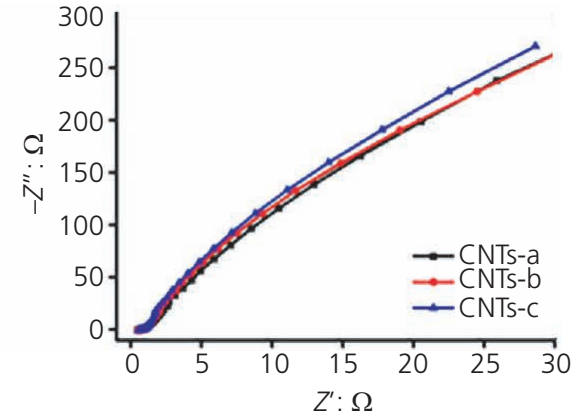

(c)

Figure 7. (a) CV curves of CNTs-a, CNTs-b and CNTs-c at a scan rate of $10 \mathrm{mV} / \mathrm{s}$; (b) GCD curves of CNTs-a, CNTs-b and CNTs-c tested at $1 \mathrm{~A} g$; (c) Nyquist plots of the EIS for the CNTs-a, CNTs-b and CNTs-c electrodes

attributed to the higher specific surface area providing more ion adsorption sites.

Figure 7(c) shows the Nyquist plots of the EIS spectra of the materials. The plot is composed of a small semicircle in the highfrequency region and a linear component in the low-frequency region. The intercept of the semicircle on the real axis at high frequency represents the equivalent series resistance $\left(R_{\mathrm{s}}\right)$, and the diameter of the semicircle corresponds to the charge-transfer resistance $\left(R_{\mathrm{ct}}\right)$ of the electrode. ${ }^{40}$ As shown in Figure $7(\mathrm{c})$, the diameters of the semicircles in all plots are very small, suggesting a negligible interfacial electron-transfer resistance on all the three materials. In other words, the introduction of pyrrole maintains the excellent conductivity of the CNT film, which is favorable for its CDI performance. In addition, the $R_{\mathrm{s}}$ of the CNTs-c electrode is merely $\sim 0.63 \Omega$, which suggests a higher charge-discharge rate and a highly efficient electrosorption behavior. Moreover, the slope of the straight line for CNTs-c is also much larger than those of CNTs-a and CNTs-b, further confirming the very facile diffusion of the salt ions onto the electrode surface, which can be attributed to the mesoporous structure caused by the pyrrole. ${ }^{40}$
Figure 8(a) depicts the electrosorptive capacities of the CNTs-a, CNTs-b and CNTs-c electrodes. Compared with the other two samples, the CNTs-c film possessed an obviously better desalination performance. Specifically, the effective deionization time of the CNTs-c electrode is only $10 \mathrm{~min}$, while the other two materials showed a larger lag by prolonging the deionization time to $20 \mathrm{~min}$, indicating that CNTs-c has higher deionization efficiency. In addition, the specific adsorption capacity of the CNTs-c film is $11.39 \pm 0.3 \mathrm{mg} / \mathrm{g}$, which is 1.68 times higher than that of CNTs-b $(6.76 \pm 0.3 \mathrm{mg} / \mathrm{g})$ and 2.5 times higher than that of CNTs-a $(4.52 \pm 0.3 \mathrm{mg} / \mathrm{g})$, respectively, clearly showing a much enhanced desalination capacity, which can be attributed to its higher specific surface area and larger pore size/volume.

To study further the CDI stability of the materials, multiple adsorption-desorption cycles were afterward carried out on the materials, with the adsorption and desorption time both set at 20 min (Figure 8(b)). As shown, the reduction in the solution conductivity of the CNTs-c electrode was always larger than those of the CNTs-a and CNTs-b electrodes in every cycle, suggesting that CNTs-c exhibits a more rapid and efficient adsorption of the salt ions. As illustrated in Figure 8(c), the capacity of the CNTs-c

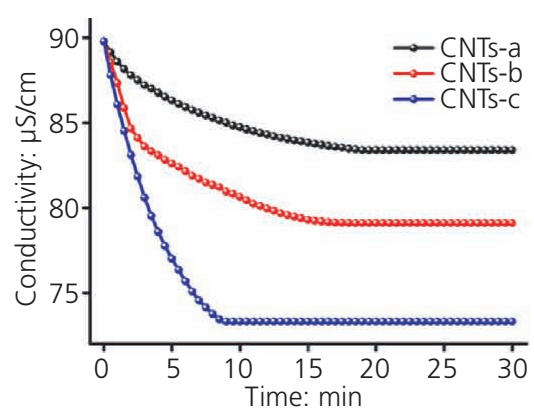

(a)

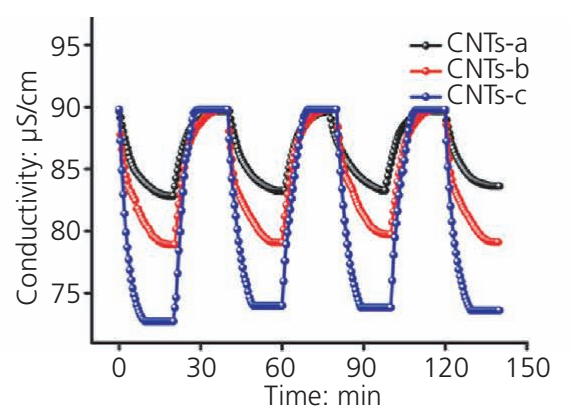

(b)

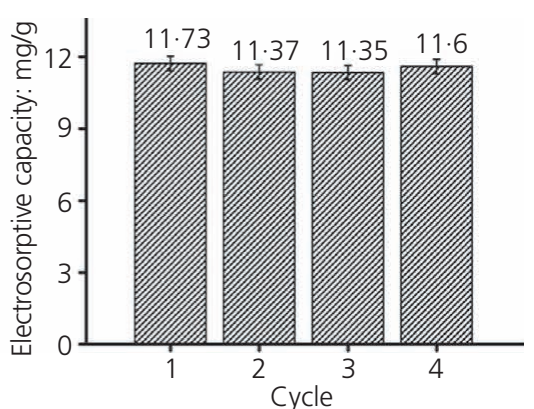

(c)

Figure 8. (a) Saturated adsorption curves of the CNTs-a, CNTs-b and CNTs-c electrodes under a working voltage of 2.0 V in sodium chloride solution; (b) adsorption-desorption curves of CNTs-a, CNTs-b and CNTs-c for four cycles; (c) capacity of CNTs-c electrode for four cycles 
electrode after each cycle is calculated to be $11 \cdot 73,11 \cdot 37,11.35$ and $11.60 \mathrm{mg} / \mathrm{g}$, corresponding to a very stable adsorption capacity in these four cycles and confirming that the CNTs-c film could be thoroughly regenerated for CDI without applying any extra driving energy, which is the key for large-scale applications.

Based on the aforementioned characterizations and performance evaluations, the best performance of the CNTs-c material can be attributed to several aspects. Firstly, when pyrrole is introduced into the precursor solution, the obtained CNTs turned from the long and straight structure in CNTs-a into a curved form in CNTs-c. This will lead to an enlarged pore size of the film, which makes the CNT films more accessible to salt ions, leading to faster CDI. Secondly, with the introduction of pyrrole, the specific surface area and pore volume could also be increased. As a result, the obtained CNT film can absorb a larger number of ions, which is crucial to achieving a higher CDI capacity. Thirdly, although relatively more defective sites exist in CNTs-c, its electronic conductivity is, however, not obviously affected, as can be evidenced from the EIS results, greatly facilitating the electrochemical CDI process on this material. These advantages thus comprehensively result in the best CDI performance on this CNTs-c material, in comparison with both CNTs-a and CNTs-b.

\section{Conclusion}

In summary, freestanding CNT films with a high specific surface area and tunable structures were synthesized by using the FCCVD method. The introduction of pyrrole in the precursor has resulted in a significant structural change and a tunable pore structure in the obtained CNT films, making them favorable for CDI application as electrode materials. For the CNTs-c film that was prepared with $2 \mathrm{wt} . \%$ of pyrrole, its specific electrosorptive capacity reached $11.39 \mathrm{mg} / \mathrm{g}$. Furthermore, the freestanding CNT film with a large specific surface area and a reasonable pore structure is suitable for the large-scale application of CDI.

\section{Acknowledgements}

This work was supported by funding from the National Natural Science Fund Program of China (Grant Number 51072130) and Open Research Fund Program of Guangdong Key Laboratory of Membrane Material and Membrane Separation, Guangzhou Institute of Advanced Technology, Chinese Academy of Sciences (Grant Number 2016032101).

\section{REFERENCES}

1. Karagiannis IC and Soldatos PG (2008) Water desalination cost literature: review and assessment. Desalination 223(1-3): 448-456.

2. Zhang D, Wen $X$ and Shi L (2012) Enhanced capacitive deionization of graphene/mesoporous carbon composites. Nanoscale 4(17): 5440-5446.

3. Hillie T and Hlophe M (2007) Nanotechnology and the challenge of clean water. Nature Nanotechnology 2(11): 663-664.

4. Elimelech M and Phillip WA (2011) The future of seawater desalination: energy, technology, and the environment. Science 333(6043): 712-717.

5. Pan SY, Snyder SW and Lin YJ (2018) Electrokinetic desalination of brackish water and associated challenges in the water and energy nexus. Environmental Science: Water Research \& Technology 4(5): 613-638.

6. Zhao R, Porada S and Biesheuvel PM (2013) Energy consumption in membrane capacitive deionization for different water recoveries and flow rates, and comparison with reverse osmosis. Desalination 330(25): 35-41.

7. Wang $Y$ and Han $X$ (2015) Preparation optimization on the coatingtype polypyrrole/carbon nanotube composite electrode for capacitive deionization. Electrochimica Acta 182: 81-88.

8. Porada S and Zhao R (2013) Review on the science and technology of water desalination by capacitive deionization. Progress in Materials Science 58(8): 1388-1442.

9. Li HB and Lu T (2009) Electrosorption behavior of graphene in $\mathrm{NaCl}$ solutions. Journal of Materials Chemistry 19(37): 6773-6779.

10. Zou L and Qi D (2008) Using activated carbon electrode in electrosorptive deionisation of brackish water. Desalination 225(1): 329-340.

11. Alencherry T and Naveen AR (2017) Effect of increasing electrical conductivity and hydrophilicity on the electrosorption capacity of activated carbon electrodes for capacitive deionization. Desalination 415: 14-19.

12. Liu X, Chen T and Qiao WC (2017) Fabrication of graphene/activated carbon nanofiber composites for high performance capacitive deionization. Journal of the Taiwan Institute of Chemical Engineers 72: 213-219.

13. Jung $\mathrm{HH}$, Hwang SW and Hyun SH (2007) Capacitive deionization characteristics of nanostructured carbon aerogel electrodes synthesized via ambient drying. Desalination 216(1-3): 377-385.

14. Wang L, Wang M and Huang ZH (2011) Capacitive deionization of $\mathrm{NaCl}$ solutions using carbon nanotube sponge electrodes. Journal of Materials Chemistry 21(45): 18295-18299.

15. Hou CH, Liu NL and Hsu HL (2014) Development of multi-walled carbon nanotube/poly (vinyl alcohol) composite as electrode for capacitive deionization. Separation and Purification Technology 130: 7-14.

16. Zhang D, Wen X and Shi L (2012) Enhanced capacitive deionization of graphene/mesoporous carbon composites. Nanoscale 4(17): 5440-5446.

17. Nugrahenny ATU, Kim J and Kim SK (2014) Preparation and application of reduced graphene oxide as the conductive material for capacitive deionization. Carbon Letters 15(1): 38-44.

18. Zhang S, Wang $Y$ and Han $X$ (2018) Optimizing the fabrication of carbon nanotube electrode for effective capacitive deionization via electrophoretic deposition strategy. Progress in Natural Science: Materials International 28(2): 251-257.

19. He Y, Chen W and Gao C (2013) An overview of carbon materials for flexible electrochemical capacitors. Nanoscale 5(19): 8799-8820.

20. Dai K, Shi LY and Fang JH (2005) NaCl adsorption in multi-walled carbon nanotubes. Materials Letters 59(16): 1989-1992.

21. Gao HX, Hou F and Zheng XR (2015) Electrochemical property studies of carbon nanotube films fabricated by CVD method as anode materials for lithium-ion battery applications. Vacuum 112: 1-4.

22. Tewari A and Sharma SC (2015) Effect of different carrier gases and their flow rates on the growth of carbon nanotubes. Physics of Plasmas 22(4): 043501.

23. Li H, Pan L and Lu T (2011) A comparative study on electrosorptive behavior of carbon nanotubes and graphene for capacitive deionization. Journal of Electroanalytical Chemistry 653(1-2): 40-44.

24. Deng C, Jiang J and Liu F (2015) Influence of carbon nanotubes coatings onto carbon fiber by oxidative treatments combined with electrophoretic deposition on interfacial properties of carbon fiber composite. Applied Surface Science 357: 1274-1280.

25. Yoon H, Lee J and Kim SR (2016) Capacitive deionization with Caalginate coated-carbon electrode for hardness control. Desalination 392: 46-53.

26. Sinnott SB and Andrews R (1999) Model of carbon nanotube growth through chemical vapor deposition. Chemical Physics Letters 315(1-2): 25-30. 
A freestanding CNTs film fabricated by pyrrole-modified CVD for capacitive deionization

Song, Guo, Zhang et al.
27. Cao A, Xu C and Liang J (2001) X-ray diffraction characterization on the alignment degree of carbon nanotubes. Chemical Physics Letters 344(1): 13-7.

28. Haniff MASM and Hafiz SM (2017) Nitrogen-doped multiwalled carbon nanotubes decorated with copper(I) oxide nanoparticles with enhanced capacitive properties. Journal of Materials Science 52(11): 6280-6290.

29. Zhou Y, Hou F and Wang ZP (2014) Facile synthesis of $\mathrm{Ni}(\mathrm{OH})_{2}$ nanoflakes on carbon nanotube films as flexible binder-free electrode for supercapacitors. ECS Solid State Letters 3(1): 177-186.

30. Yang SB and Feng XL (2011) Graphene-based carbon nitride nanosheets as efficient metal-free electrocatalysts for oxygen reduction reactions. Angewandte Chemie International Edition 50(23): 5339-5343.

31. Liang J and Zhou RF (2014) Fe-N decorated hybrids of CNTs grown on hierarchically porous carbon for high-performance oxygen reduction. Advanced Materials 26(35): 6074-6079.

32. Yu Q, Guan D and Zhuang Z (2017) Mass production of monodisperse carbon microspheres with size-dependent supercapacitor performance via aqueous self-catalyzed polymerization. ChemPlusChem 82(6): 872-878.

33. Shen G, Sun X and Zhang H (2015) Nitrogen-doped ordered mesoporous carbon single crystals: aqueous organic-organic selfassembly and superior supercapacitor performance. Journal of Materials Chemistry A 3(47): 24041-24048.
34. Sun $X$, Zhang $H$, Zhou L and Huang X (2016) Polypyrrole-coated zinc ferrite hollow spheres with improved cycling stability for lithium-ion batteries. Small 12(27): 3732-3737.

35. Yang S and Chang K (2011) Design and tailoring of a hierarchical graphene-carbon nanotube architecture for supercapacitors. Journal of Materials Chemistry 21(7): 2374-2380.

36. Sui $Z$ and Meng X (2012) Green synthesis of carbon nanotube-graphene hybrid aerogels and their use as versatile agents for water purification. Journal of Materials Chemistry 22(18): 8767-8771.

37. Lv W, Tang DM and He YB (2009) Low-temperature exfoliated graphenes: vacuum-promoted exfoliation and electrochemical energy storage. ACS Nano 3(11): 3730-3736.

38. Zhang CM and Wang Y (2018) Optimizing the fabrication of carbon nanotube electrode for effective capacitive deionization via electrophoretic deposition strategy. Progress in Natural Science: Materials International 28: 251-257.

39. Ye S and Nguyen DCT (2017) High performance for electric doublelayer capacitors based on CNT-CG composite synthesized as additive material by CVD method. Journal of Industrial and Engineering Chemistry 54: 428-433.

40. Gu XY and Yang Y (2014) Nitrogen-doped graphene composites as efficient electrodes with enhanced capacitive deionization performance. RSC Advances 4: 63189-63199.

\section{How can you contribute?}

To discuss this paper, please submit up to 500 words to the journal office at journals@ice.org.uk. Your contribution will be forwarded to the author(s) for a reply and, if considered appropriate by the editor-in-chief, it will be published as a discussion in a future issue of the journal.

ICE Science journals rely entirely on contributions from the field of materials science and engineering. Information about how to submit your paper online is available at www.icevirtuallibrary.com/page/authors, where you will also find detailed author guidelines. 